\title{
Interleukin-1 receptor-associated kinase 3 downregulation in peripheral blood mononuclear cells attenuates immunosuppression in sepsis
}

\author{
QIN XIA ${ }^{1}$, YUPIN ZHOU $^{2}, \mathrm{XI} \mathrm{WANG}^{1}$ and SHUKUN FU ${ }^{1}$ \\ ${ }^{1}$ Department of Anesthesiology, The Tenth People's Hospital of Tongji University, Shanghai 200072; \\ ${ }^{2}$ Department of Anesthesiology, Shanghai Dermatology Hospital, Shanghai 200050, P.R. China
}

Received February 22, 2017; Accepted September 13, 2017

DOI: $10.3892 / \mathrm{etm} .2017 .5549$

\begin{abstract}
Sepsis is the leading cause of mortality in intensive care units due to complex inflammatory immune responses and immunosuppression. Recent studies have indicated that the negative regulator of toll like receptors, interleukin-1 receptor-associated kinase 3 (IRAK-3/IRAK-M), serves an important role in immunosuppression during sepsis. In the current study, a cecal ligation puncture model was established in mice using lipopolysaccharide secondary challenge to simulate immunosuppression in sepsis. Peripheral blood mononuclear cells (PBMCs) from this model were then used to evaluate the expression and function of IRAK-M. The results demonstrated that silencing of IRAK-M expression in PBMCs from immunosuppressed mice partially restored the production of pro-inflammatory cytokines. By introducing PBMCs transfected with small-interfering RNA targeting IRAK-M into septic immunosuppressed mice, the survival rate was improved with an increase in splenic $\mathrm{CD}^{+}$and $\mathrm{CD}^{+} \mathrm{T}$ cells and a decrease in $\mathrm{T}$ cell apoptosis. In conclusion, downregulation of IRAK-M reversed the effects of sepsis on the production of inflammatory cytokines in PBMCs, and improved the survival of septic immunosuppressed mice. These results provide a basis for future studies investigating the immunological mechanisms underlying immune suppression in sepsis.
\end{abstract}

\section{Introduction}

Sepsis is a complex systemic inflammatory immune response associated with organ dysfunction caused by trauma or infection with pathogens. It is the most common cause of

Correspondence to: Professor Shukun Fu, Department of Anesthesiology, The Tenth People's Hospital of Tongji University, 301 Middle Yanchang Road, Shanghai 200072, P.R. China

E-mail: fushukun@yeah.net

Key words: sepsis, interleukin-1 receptor-associated kinase 3, immune suppression mortality in intensive care units in the USA (1), and early studies suggest that the uncontrolled, sustained upregulation of systemic inflammatory responses is the primary underlying cause (1-3). However, subsequent results have demonstrated that the anti-inflammatory mechanisms induced by inflammatory feedback loops leads to a more complex phase of immunosuppression known as immune paralysis, which is currently considered to be the major pathological reason underlying the high mortality rates in patients with sepsis (2). Therefore, investigating the molecular mechanisms of immunosuppression may provide novel insights for the development of immune modulation therapy to treat patients with sepsis.

Interleukin-1 receptor-associated kinase 3 (IRAK-3/ IRAK-M) is an important negative regulator of toll-like receptors (TLRs) (3). It was originally thought that IRAK-M was expressed in monocytes, macrophages and other immune cells alone (4). However, subsequent studies have demonstrated that epithelial cells, including alveolar and bronchial epithelial cells, also express IRAK-M (3). IRAK-M negatively regulates TLR signaling pathways via the TGF- $\beta$-activated kinase 1 (TAK1)-dependent and mitogen-activated protein kinase kinase (MEKK)-dependent signaling pathways $(3,4)$. IRAK-M downregulates TLRs via the TAK1-dependent signaling pathway by inhibiting the formation of downstream IRAK-1/2-TNF receptor-associated factor 6 complexes via inhibition of IRAK-1 phosphorylation. This prevents de-polymerization of the IRAK-1/2 complex and inhibits the subsequent signaling cascade $(3,5-7)$. IRAK-M functions to negatively regulate TLRs via the MEKK-dependent signaling pathway by interacting with myeloid differentiation factor 88 and IRAK-4 to induce the expression of downstream negative regulators, including suppressor of cytokine signaling 1 , SH2-containing inositol phosphatase 1, protein A20 and $\mathrm{I} \kappa \mathrm{B} \alpha(4,6)$. Stimulation of monocytes isolated from patients with sepsis with lipopolysaccharide (LPS) has been associated with an increase in IRAK-M expression, and cells established a hypo-responsive status similar to immunosuppression (8).

A previous study involving a mouse model of sepsis, demonstrated that downregulation of IRAK-M in macrophages was associated with elevated survival and bacterial clearance rates in LPS-induced septic mice (9). A number of previous studies have investigated the function of IRAK-M in 
LPS-induced models; however, these studies have established models of endotoxin tolerance $(10,11)$. These models are limited, as they do not accurately represent the genuine status of a patient with multiple bacterial infections, nor the immune response to sepsis under clinical conditions (9). Therefore, a mouse cecal ligation puncture model (CLP) was employed in the present study, as it is a commonly used model of sepsis that is used to represent the pathological conditions of sepsis (9). The model was established using LPS challenge following CLP to investigate the function of IRAK-M under septic immunosuppression conditions. Peripheral blood mononuclear cells (PBMCs) were collected and IRAK-M expression was altered by transfection with small interfering RNA (siRNA).

\section{Materials and methods}

Animals. A total of 60 C57BL/6 mice (age, 8-10 weeks; 30 male and 30 female mice; weight, $25.4 \pm 2.2 \mathrm{~g}$ ) were purchased and underwent CLP surgery to induce polymicrobial sepsis. Mice were first anesthetized and an abdominal incision was made. The cecum was mobilized, ligated and then punctured once with a 22-gauge needle. The abdomen was closed by layers. Sham-operated mice underwent the same procedure but without ligation and puncture. Following surgery, $1 \mathrm{ml}$ normal saline was used for fluid resuscitation. Mice were allowed free access to food and water in temperature-controlled specific pathogen free (SPF) animal houses $\left(25^{\circ} \mathrm{C}, 50-60 \%\right.$ humidity) with access to a 12-h light/dark cycle. Septic immunosuppression status was achieved by intraperitoneal injection of $10 \mathrm{mg} / \mathrm{kg}$ purified Escherichia coli LPS (serotype 0111:B4; Sigma-Aldrich; Merck KGaA, Darmstadt, Germany) at 3 days following CLP surgery (Fig. 1), mice in the sham group were also injected with the same quantity of LPS as the CLP+LPS group (6 mice per group). All experiments were approved by the Institutional Animal Care and Use Committee of Tongji University (Shanghai, China).

Isolation of PBMCs and culture conditions. Blood $(0.6 \mathrm{ml})$ was collected from the postocular venous plexus of mice and centrifuged $\left(16,000 \mathrm{x}\right.$ g for $15 \mathrm{~min}$ at $\left.4^{\circ} \mathrm{C}\right)$. Lymphocyte separation medium (PAA Laboratories; GE Healthcare, Chicago, IL, USA) was used to obtain the PBMCs according to the manufacturer's instructions. PBMCs were then centrifuged ( $300 \mathrm{x} \mathrm{g}$ for $5 \mathrm{~min}$ at $4^{\circ} \mathrm{C}$ ) and resuspended in RPMI 1640 culture medium (Gibco; Thermo Fisher Scientific, Inc., Waltham, MA, USA) containing $10 \%$ fetal bovine serum (Gibco; Thermo Fisher Scientific, Inc.) Cells were subsequently stimulated with $50 \mathrm{ng} / \mathrm{ml}$ phorbol 12-myristate 13-acetate (Sigma-Aldrich; Merck KGaA), $500 \mathrm{ng} / \mathrm{ml}$ ionomycin (Abcam, Cambridge, MA, USA) and $3 \mathrm{mg} / \mathrm{ml}$ Brefeldin A (Sigma-Aldrich; Merck $\mathrm{KGaA}$ ) for $5 \mathrm{~h}$ prior to further experiments.

ELISAs. The PBMC culture medium was centrifuged at 3,000 $\mathrm{rpm}\left(\right.$ at $4^{\circ} \mathrm{C}$ for $5 \mathrm{~min}$ ) and the supernatant were collected to determine cytokine levels. Mouse blood $(0.6 \mathrm{ml})$ was harvested from the postocular venous plexus and serum was obtained for cytokine measurement. Mouse tumor necrosis factor- $\alpha$ (TNF- $\alpha$ ) (cat. no. 900-K54), interleukin (IL)-6 (cat. no. 900-K50) and IL-10 ELISA kits (cat. no. 900-K21) (PeproTech, Inc., Rocky Hill, NJ, USA) were used according to the manufacturer's instructions, and the samples were read using a microplate reader at a wavelength of $450 \mathrm{~nm}$. A total of $\geq 3$ replicates were included for each experiment involving the analysis of PBMCs in vitro.

Transfection of PBMCs in vitro and generation of in vivo transfer model. Mouse PBMCs $\left(1 \times 10^{6}\right)$ were transfected with $1 \mu \mathrm{g}$ IRAK-M siRNA or control siRNA (Shanghai GenePharma Co., Ltd., Shanghai, China) using Lipofectamine 2000 (Invitrogen; Thermo Fisher Scientific, Inc.) according to the manufacturer's protocol. The following target sequences were used: Murine IRAK-M forward, 5'-TGTCCCAAGTATTCC AGTA-3' and reverse, 5'-GTCCTACTGTGATCAGTTT-3'. Gene expression and cytokine production were analyzed at $24 \mathrm{~h}$ following transfection. PBMCs transfected with IRAK-M siRNA or control siRNA were also administered to mice. Following CLP surgery and LPS challenge, C57BL/6 mice were divided into four groups at random ( $n=6 /$ group) and injected intravenously with control-PBMCs or si-PBMCs (1x10 ${ }^{6}$ cells/mouse, 2 days postoperatively). The following grousp were established: Sham+LPS group; CLP+LPS group, no treatment with PMBCs; CLP-LPS-si group, treated with PMBCs transfected with IRAK-M siRNA; and CLP-LPS-con group, treated with PMBCs transfected with control siRNA). The survival rate was recorded every $24 \mathrm{~h}$. Mouse blood samples and splenocytes were obtained by grinding the spleen and lysed via RBC lysis at $24 \mathrm{~h}$ following administration of transfected PBMCs (Fig. 1). Mice that succumbed within one day following injection of PBMCs were included in the survival rate calculation but excluded from the analysis of blood and splenocyte samples, as well as further measurements.

Flow cytometry and apoptosis analysis. Anti-mouse-CD4allophycocyanin (APC) (cat. no. MHCD0405), anti-mouseCD8-APC (cat. no. 47-0081-82) and Alexa-Fluor-488Annexin V-FITC (cat. no. BMS147FI) were purchased from eBiosciences (Invitrogen; Thermo Fisher Scientific, Inc., USA). Mouse splenocytes were prepared in PBS and stained with antibodies at $4^{\circ} \mathrm{C}$ for $15 \mathrm{~min}$ at a dilution of 1:50,000. Following three washes with washing buffer $(3 \mathrm{~min}$ for each time), cells were resuspended in washing buffer (eBioscience; Thermo Fisher Scientific, Inc.) and analyzed. Fluorescence data from 10,000 lymphocyte events/sample were obtained using a FACS BD LSR II flow cytometer (BD Biosciences) and analyzed using FlowJo software 10.2 (FlowJo LLC, Ashland, OR, USA).

Reverse transcription-quantitative polymerase chain reaction ( $q P C R)$. Total RNA was extracted from PBMCs using TRIzol ${ }^{\circledR}$ reagent (Invitrogen; Thermo Fisher Scientific, Inc., USA) and reverse transcript with PrimeScript ${ }^{\mathrm{TM}}$ RT Master mix (Takara, Otsu, Japan) according to the manufacturer's instructions. qPCR analysis was performed using a 7900HT Fast Real-time PCR system and SYBR-Green Real-Time PCR Master Mixes (Thermo Fisher Scientific, Inc.) and SYBR-Green dye. The folliwowing thermocycling conditions were used: $94^{\circ} \mathrm{C}$ for $30 \mathrm{sec}, 59^{\circ} \mathrm{C}$ for $45 \mathrm{sec}$ and $72^{\circ} \mathrm{C}$ for $60 \mathrm{sec}$ for a total of 35 cycles. Target gene expression was normalized to that of GAPDH forward, 5'-GAGAGTGTTTCCTCGTCCCGTAG-3' and reverse, 5'-GCCTCACCCCATTTGATGTTAGT-3'. The 


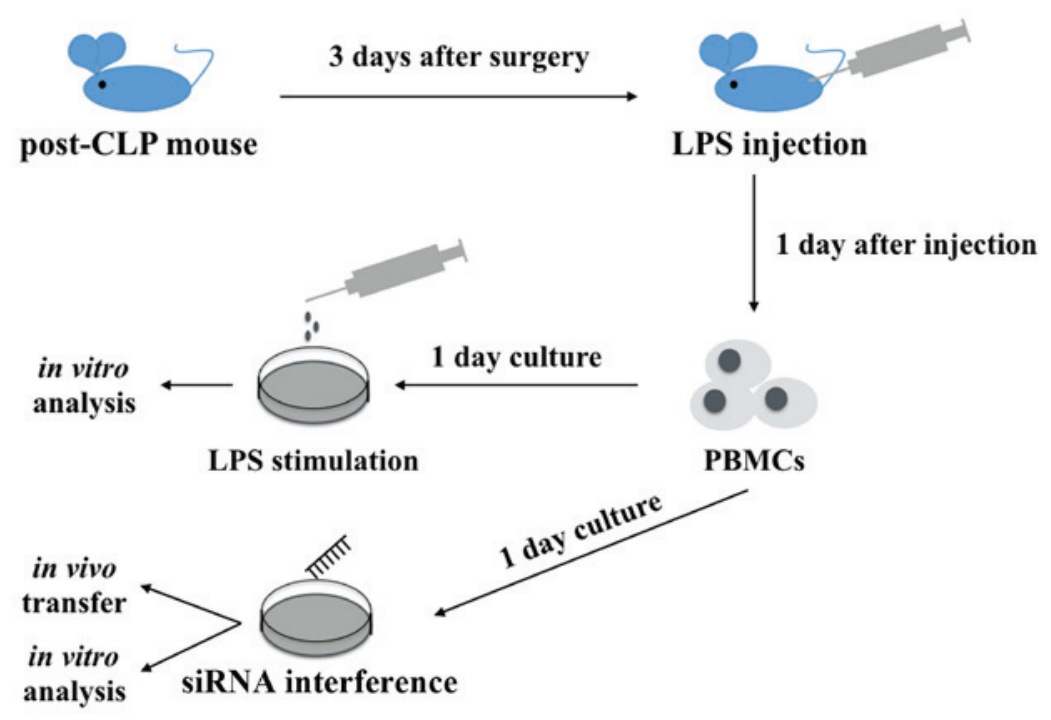

Figure 1. Study design. C57BL/6 mice underwent CLP surgery and $1 \mathrm{ml}$ normal saline was injected intraperitoneally for fluid resuscitation. At 3 days following surgery, the post-CLP mice were intravenously injected with LPS. PBMCs were harvested 1 day following LPS injection and then cultured for $24 \mathrm{~h}$. PBMCs were stimulated with LPS for further analysis or transfected with siRNA for downstream experiments. CLP, cecal ligation puncture procedure; LPS, lipopolysaccharide; PBMCs, peripheral mononuclear blood cells; siRNA, small interfering RNA.

following primer sets were used: IRAK-M forward, 5'-ACG TCACTGAAGGCACTGAG-3' and reverse, 5'-TTTGACTGA GAGGCGGTCAC-3'. Analysis of relative gene expression was performed using qPCR and the $2^{-\Delta \Delta \mathrm{Cq}}$ method (12).

Western blot analysis. BCA assay was performed to determine protein concentrations. Protein extraction was performed using radioimmunoprecipitation assay (Beyotime Institute of Biotechnology). A total of $40 \mu \mathrm{g}$ of separated protein from samples (PBMCs transfected with si-IRAK-M or si-controls) were loaded per lane and subjected to $10 \%$ SDS-PAGE (IRAK-M molecular weight, $68 \mathrm{kDa}$ ). Subsequently, the samples were then transferred onto a polyvinylidene difluoride membrane (Merck KGaA) using conventional methods. Membranes were blocked using 5\% non-fat milk, at room temperature for $1 \mathrm{~h}$. Following this, membranes were incubated with anti-IRAK-M (dilution, 1:1,000; cat. no. 4369T) and anti-GAPDH (dilution, 1:1,000; cat. no. 5174T; both from Cell Signaling Technology, Inc., Danvers, MA, USA) at $4^{\circ} \mathrm{C}$ overnight. Subsequently, blots were exposed to peroxidase-conjugated goat anti-rabbit $\operatorname{IgG}$ secondary antibody (dilution, 1:5,000; cat. no. 5172; Yeasen, Shanghai, China) at room temperature for $1 \mathrm{~h}$ and visualized using a ECL Plus kit (Beyotime Institute of Biotechnology, Haimen, China).

Statistical analysis. All measurements were repeated in triplicate. Data were statistically analyzed using a Student's t-test or one-way analysis of variance. SPSS software 16.0 (SPSS, Inc., Chicago, IL, USA) was used for statistical analyses. $\mathrm{P}<0.05$ was determined to indicate a statistically significant difference.

\section{Results}

PBMCs derived from a immunosuppression mouse model of sepsis exhibit a decrease in the production of pro-inflammatory cytokines and immunosuppression. To evaluate the immunosuppression status of PBMCs, mice were intraperitoneally injected with LPS at 3 days following CLP surgery, and PBMCs were collected from blood samples. The control group consisted of mice that had undergone sham CLP surgery followed by injection with the same quantity of LPS. PBMCs were isolated from mice at 1 day following LPS injection, and were subsequently cultured in the presence of LPS for $24 \mathrm{~h}$ to evaluate cytokine production. When compared with the controls, the levels of TNF- $\alpha$ and IL-6 pro-inflammatory cytokines were significantly decreased, whereas IL-10 regulatory cytokine levels were significantly increased in the CLP+LPS group (all $\mathrm{P}<0.05$; Fig. 2A-C). This indicated that cells were refractory to LPS stimulation, as they exhibited an inflammatory-suppressed phenotype. The expression of IRAK-M in PBMCs was subsequently analyzed, and the results indicated a significant increase in IRAK-M expression in PBMCs derived from CLP+LPS mice when compared with those isolated from control group mice $(\mathrm{P}<0.05$; Fig. 2D). This demonstrates that PBMCs from CLP+LPS mice display an immunosuppressed status and are refractory to further LPS stimulation.

IRAK-M downregulation partially reverses cytokine production in immunosuppressed PBMCs. To investigate whether IRAK-M influences cytokine production, siRNAs targeting IRAK-M were transfected into PBMCs to inhibit IRAK-M expression. As demonstrated in Fig. $3 \mathrm{~A}$ and $\mathrm{B}$, the level of IRAK-M mRNA $(\mathrm{P}<0.05)$ and protein was decreased in the IRAK-M siRNA group when compared with the control group. The cytokine levels in the culture medium were then analyzed. Downregulation of IRAK-M resulted in a significant increase in TNF- $\alpha$ and IL- 6 and a significant decrease in IL-10 production when compared with the control group (all $\mathrm{P}<0.05$; Fig. 3C-E). This indicated that downregulation of IRAK-M reversed the effects of CLP and LPS on cytokine production. Therefore, downregulating the expression of IRAK-M may allow immunosuppressed PBMCs to partially regain their pro-inflammatory features and cytokine production levels. 

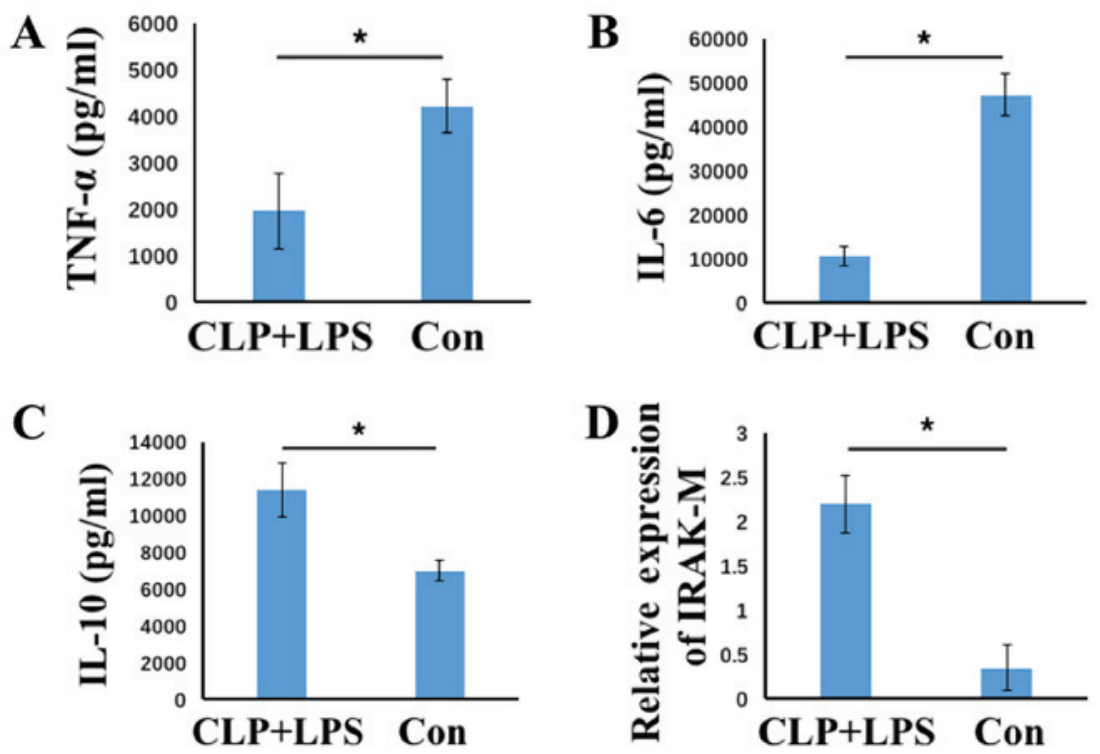

Figure 2. Expression of TNF- $\alpha$, IL-6, IL-10 and IRAK-M in CLP+LPS and control mice. The concentration of (A) TNF- $\alpha$, (B) IL-6 and (C) IL-10 in the supernatant of the culture medium of PBMCs was analyzed using ELISAs, and (D) IRAK-M expression was analyzed using reverse transcription-quantitative polymerase chain reaction in PBMCs from CLP-LPS mice $(n=6)$ or control mice $(n=6)$. The results are presented as the mean \pm standard deviation. ${ }^{*} \mathrm{P}<0.05$ as indicated. CLP, cecal ligation puncture procedure; LPS, lipopolysaccharide; TNF- $\alpha$, tumor necrosis factor- $\alpha$; IL, interleukin; IRAK-M, interleukin- 1 receptor-associated kinase 3; CLP-LPS, mice that underwent CLP surgery followed by LPS challenge; con, mice that underwent sham surgery followed by LPS challenge.

\section{A}
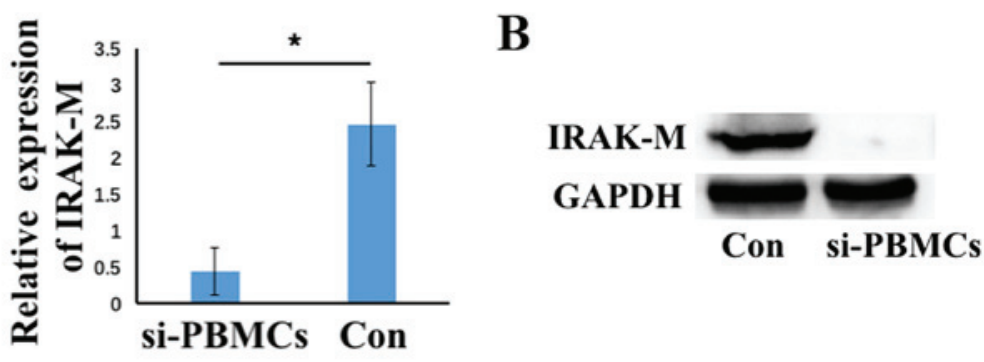

C

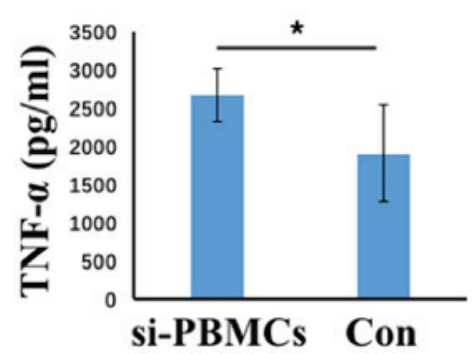

D

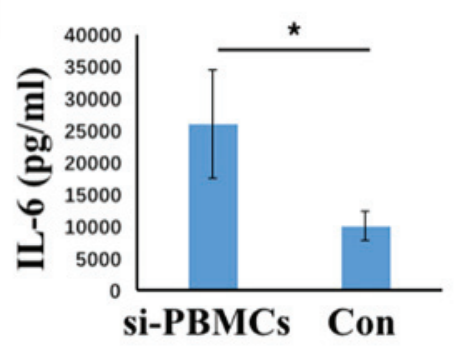

E

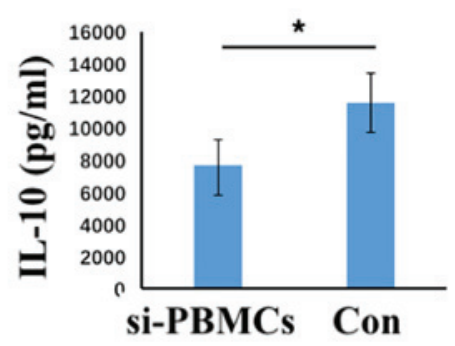

Figure 3. Expression of TNF- $\alpha$, IL-6, IL-10 and IRAK-M following IRAK-M downregulation in PMBCs. The level of IRAK-M (A) mRNA and (B) protein was analyzed using reverse transcription-polymerase chain reaction and western blotting analyses, respectively, following transfection of PMBCs with IRAK-M or control siRNA. The concentration of (C) TNF- $\alpha$, (D) IL-6 and (E) IL-10 in the supernatant of culture medium following transfection with siRNA was analyzed using ELISAs. Data are presented as the mean \pm standard deviation $(n=6)$. * $\mathrm{P}<0.05$ as indicated. TNF- $\alpha$, tumor necrosis factor- $\alpha$; IL, interleukin; IRAK-M, interleukin-1 receptor-associated kinase 3; PMBCs, peripheral mononuclear blood cells; siRNA, small interfering RNA; CLP, cecal ligation puncture; si-PMBCs, PMBCs derived from CLP-LPS mice transfected with IRAK-M-siRNA; con, PMBCs from CLP-LPS mice transfected with control-siRNA. 

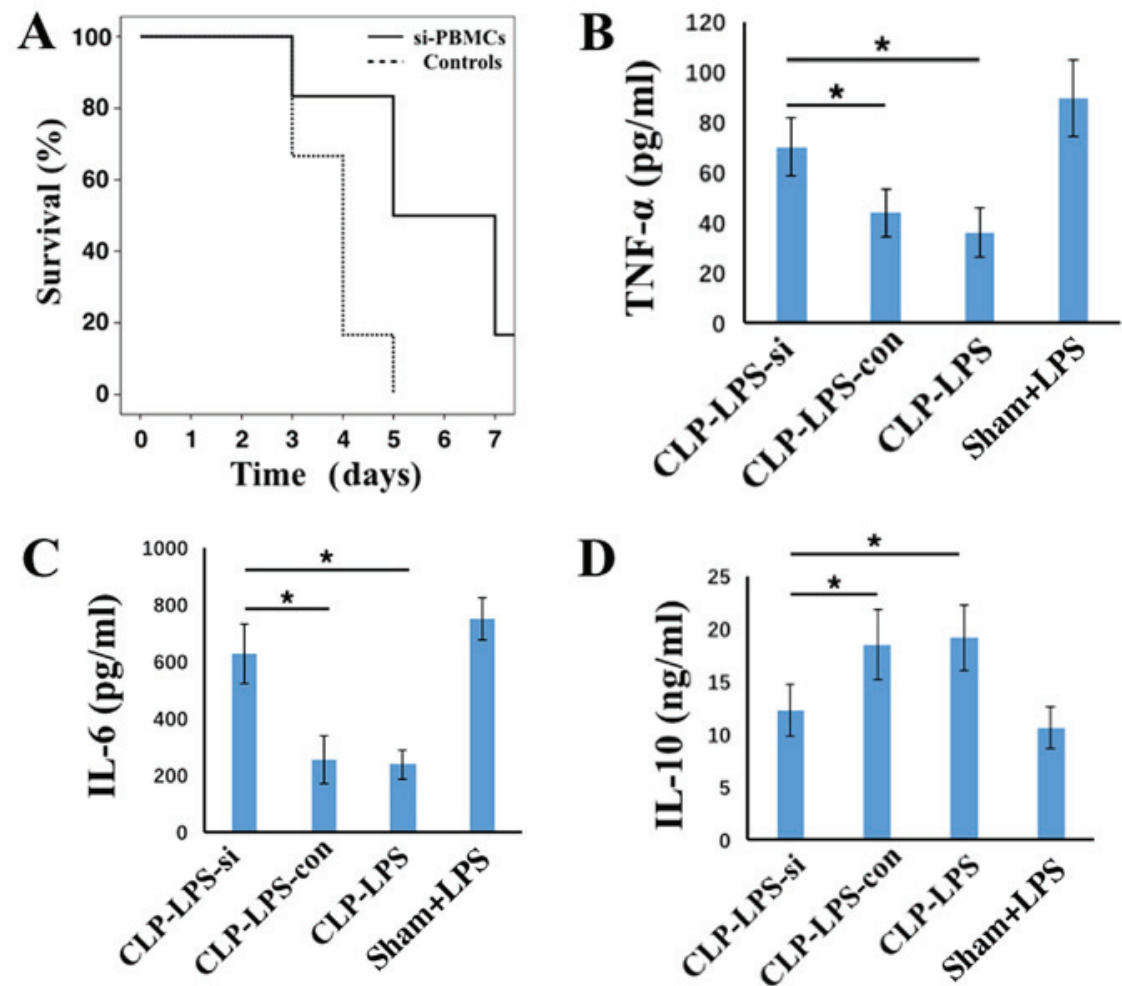

Figure 4. Survival rate and serum TNF- $\alpha$, IL-6 and IL-10 levels in mice administered with PBMCs transfected with IRAK-M siRNA. (A) The survival rate and level of (B) TNF- $\alpha$, (C) IL-6 and (D) IL-10 levels in the serum of CLP-LPS mice injected with PBMCs transfected with IRAK-M siRNA (n=6) or control siRNA $(\mathrm{n}=6)$ compared with the sham+LPS and CLP+LPS mice that were not injected with PBMCs. The results are presented as the mean \pm standard deviation. " P<0.05 as indicated. TNF- $\alpha$, tumor necrosis factor- $\alpha$; IL, interleukin; IRAK-M, interleukin-1 receptor-associated kinase 3; PMBCs, peripheral mononuclear blood cells; siRNA, small interfering RNA; CLP, cecal ligation puncture procedure; LPS, lipopolysaccharide; CLP-LPS-si, mice that underwent CLP surgery followed by LPS challenge and injection with PBMCs transfected with IRAK-M siRNA; CLP-LPS-con, mice that underwent CLP surgery followed by LPS challenge and injection of PMBCs transfected with control siRNA; CLP-LPS, mice that underwent CLP surgery followed by LPS challenge; Sham+LPS, mice that underwent sham surgery followed by LPS challenge only.

Downregulation of IRAK-M in PBMCs leads to immunosuppression and improves the survival of septic mice. Following the observation that a reduction in IRAK-M expression partially recovered the pro-inflammatory phenotype of PBMCs, further experiments were performed to investigate the survival rate and level of immunosuppression in septic mice treated with transfected PMBCs. The CLP+LPS model was used to simulate the immunosuppression phase of sepsis, and PBMCs transfected with IRAK-M or control siRNA were intravenously injected into these mice. Mouse survival rates and serum cytokine levels were subsequently monitored. As demonstrated in Fig. 4A, the survival time and rate were increased in mice treated with IRAK-M-downregulated PBMCs when compared with those transfected with control siRNAs. At 1 day following injection of PBMCs (equivalent to 5 days following surgery), TNF- $\alpha$ and IL-6 levels were significantly increased and IL-10 levels were significantly decreased in CLP+LPS group mice injected with PBMCs transfected with IRAK-M siRNA when compared with CLP+LPS mice treated with PBMCs transfected with control siRNA (all $\mathrm{P}<0.05$; Fig. 4B-D). Mice treated with IRAK-M-downregulated PBMCs remained in a septic state. These results indicate that treatment with PBMCs improved the immune and inflammatory responses of mice with sepsis.

Silencing of IRAK-M in PBMCs activates and decreases apoptosis of splenic $T$ cells. To further analyze the immune status of mice treated with IRAK-M-silenced PBMCs, their splenocytes were harvested in order to determine the number of $\mathrm{CD}^{+}$and $\mathrm{CD} 8^{+} \mathrm{T}$ cells and their rate of apoptosis. Cells were double-stained with annexin V and anti-CD4 or anti-CD8 antibodies. A demonstrated in Fig. 5, the number of splenic $\mathrm{CD}^{+}$and $\mathrm{CD} 8^{+} \mathrm{T}$ cells was significantly increased in the CLP+LPS group treated with IRAK-M-silenced PBMCs when compared with the CLP+LPS control siRNA-treated and CLP+LPS groups (all $\mathrm{P}<0.05$; Fig. 5A-D). In addition, the number of apoptotic $\mathrm{CD} 4^{+}$or $\mathrm{CD} 8^{+} \mathrm{T}$ cells was decreased in the CLP+LPS group treated with IRAK-M-silenced PBMCs when compared with the CLP+LPS control siRNA-treated and CLP+LPS groups (all P<0.05; Fig. 5A, B, E and F). Therefore, the survival rates and serum cytokine levels indicated that treating immunosuppressed septic mice with IRAK-M-downregulated PBMCs may improve their survival and immune responses. This suggests that IRAK-M serves an important role in immune-regulation, and demonstrates its potential application in immune therapy for treating patients with sepsis.

\section{Discussion}

Sepsis is a complex systemic disease with symptoms including multiple organ dysfunction, uncontrolled inflammation, as well as cellular and molecular dysfunction (1). It has been demonstrated that, pro- and anti-inflammatory responses 

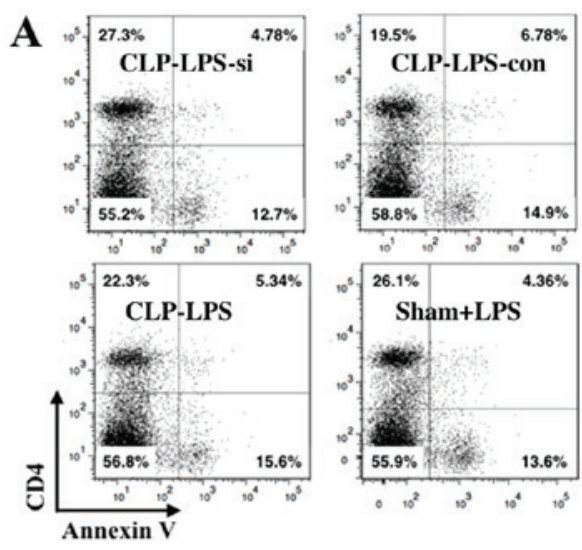

C

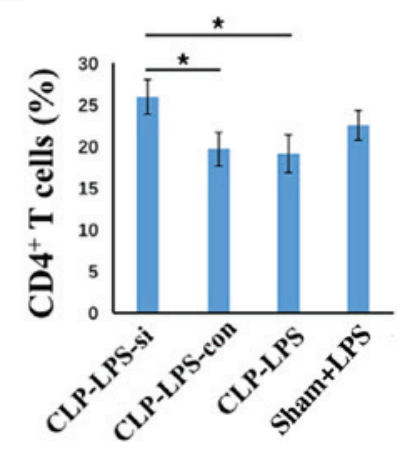

$\mathbf{E}$

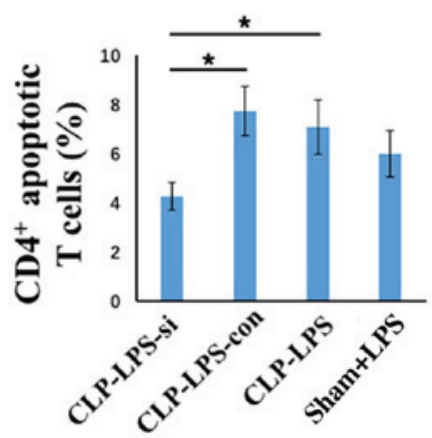

B
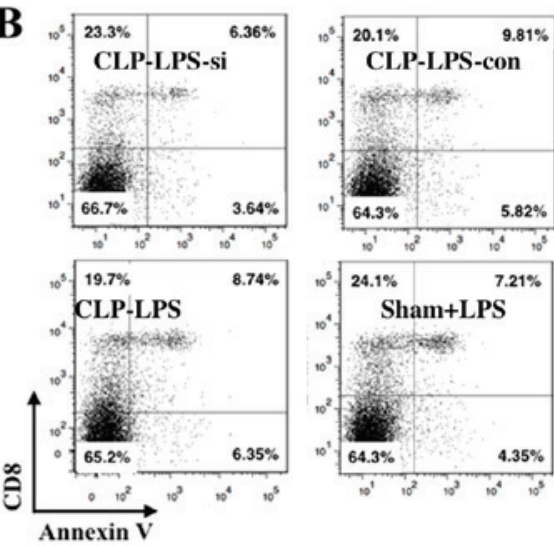

D

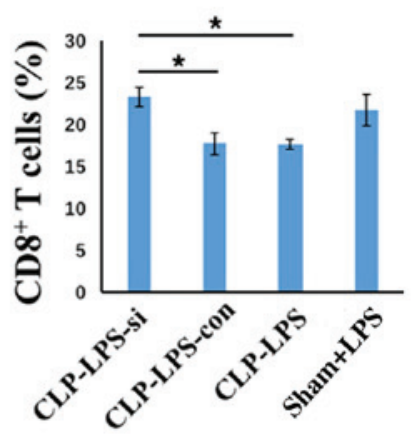

$\mathbf{F}$

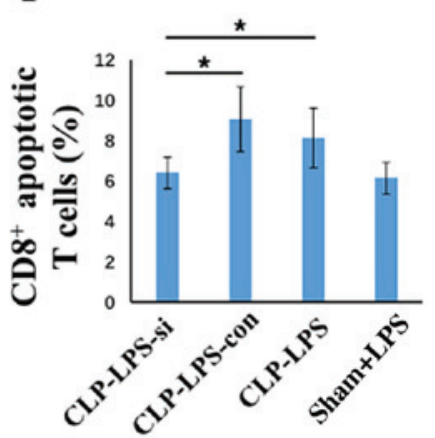

Figure 5. Treatment of septic rats with PBMCs transfected with IRAK-M siRNA led to T cell activation and a decrease in apoptosis. (A and B) Representative flow cytometry plots showing the percentage of $(\mathrm{C}) \mathrm{CD}^{+}$, (D) $\mathrm{CD} 8^{+}$, (E) apoptotic $\mathrm{CD} 4^{+}$and (F) apoptotic $\mathrm{CD} 8^{+} \mathrm{T}$ cells in CLP+LPS mice administered with IRAK-M-downregulated PBMCs ( $n=6)$, CLP+LPS mice administered with control PBMCs $(n=6), C L P+L P S$ mice ( $n=6)$ and Sham+LPS mice ( $n=6)$. The results are presented as the mean \pm standard deviation. " $\mathrm{P}<0.05$, as indicated. PMBCs, peripheral mononuclear blood cells; IRAK-M, interleukin-1 receptor-associated kinase 3; siRNA, small interfering RNA; CLP, cecal ligation puncture procedure; LPS, lipopolysaccharide; CLP-LPS-si, mice that underwent CLP surgery followed by LPS challenge and injection with PBMCs transfected with IRAK-M siRNA; CLP-LPS-con, mice that underwent CLP surgery followed by LPS challenge and injection of PMBCs transfected with control siRNA; CLP-LPS, mice that underwent CLP surgery followed by LPS challenge; Sham+LPS, mice that underwent sham surgery followed by LPS challenge only.

occur during sepsis; the balance of which may influence disease pathogenesis and patient prognosis (1). Previous treatments and research have focused on targeting the uncontrolled and amplified systemic inflammatory response during sepsis, and several strategies that aim to reduce inflammation have been proposed, including neutralizing endotoxins or inhibiting TLR signaling $(13,14)$. However, these treatments rarely improve survival rates as inhibited immune responses to pathogens promotes infection and thus worsen the clinical outcome $(13,14)$. Previous studies have also suggested that immunosuppression (also known as immune-paralysis), which results from uncontrolled anti-inflammatory feedback loops, is the primary reason for the development of secondary infections during the late phase of sepsis. This leads to more severe infections, multiple organ failure and mortality $(2,15-18)$. The key features of immunosuppression include a lack of responsiveness to inflammatory stimulation, enhanced production of anti-inflammatory cytokines and apoptosis, decreased $\mathrm{T}$ cell or dendritic cell viability, refraction of immune cells to infection or endotoxins and decreased expression of HLA-DR on monocytes (18). In the present study, post-CLP mice were injected with LPS at 3 days following surgery, and their PBMCs exhibited an immunosuppressive state with a limited response to LPS, decreased pro-inflammatory cytokine 
production, and elevated IL-10 and IRAK-M expression. Further in vitro experiments indicated that downregulating the expression of IRAK-M partially reversed these effects on cytokine production. Therefore, the results suggest that IRAK-M serves a key role in the anti-inflammatory response, which is consistent with those of a previous study demonstrating that IRAK-M is a negative regulator of inflammatory responses (19).

Previous studies have investigated the function of IRAK-M in regulating the immune response, particularly during endotoxin tolerance, which shares a number of characteristics with septic immunosuppression, such as irresponsiveness of cytokine production to stimuli and increased level of anti-inflammatory cytokines $(8,9)$. It has been demonstrated that knockout of IRAK-M in macrophages is associated with a notable increase in responsiveness to LPS following pre-treatment, as well as endotoxin tolerance (10). A further study also reported an improvement in survival rate and bacterial clearance following IRAK-M downregulation in LPS-induced septic mice (9).

The current study aimed to investigate the effects of IRAK-M downregulation in a mouse of model of sepsis involving CLP combined with LPS secondary challenge. The CLP+LPS model has been proposed to simulate the septic immunosuppression status (20). In the present study, the administration of PBMCs transfected with IRAK-M siRNA to CLP+LPS mice, was associated with an improved survival rate and serum pro-inflammatory cytokine production. To further evaluate the immunological status of these mice, splenic $\mathrm{CD}^{+}$and $\mathrm{CD} 8^{+} \mathrm{T}$ cells were also analyzed. As septic immunosuppression involves $\mathrm{T}$ cell apoptosis and non-responsiveness (21), the level of CD4 ${ }^{+}$ and $\mathrm{CD}^{+} \mathrm{T}$ cell apoptosis was also monitored. The injection of mice with PBMCs transfected with IRAK-M siRNAs was associated with activation of splenic $\mathrm{CD} 4^{+}$and $\mathrm{CD} 8^{+} \mathrm{T}$ cells, as demonstrated by the observed $\sim 10$ and $\sim 5 \%$ increase in the percentage of these cells, respectively, when compared with the other groups. In addition, the proportion of apoptotic $\mathrm{CD} 4^{+}$ and $\mathrm{CD} 8^{+} \mathrm{T}$ cells was decreased in CLP+LPS mice following the administration of PBMCs transfected with IRAK-M siRNA. The results indicate that IRAK-M-downregulated PBMCs increased survival and cytokine production, thereby re-establishing the pro-inflammatory response and enhancing splenic $\mathrm{T}$ cell viability. These modified PBMCs may therefore improve the immune response during sepsis. However, further studies that focus on dendritic cell function, bacterial clearance and inflammatory infiltration in different organs will be required to investigate immune status following administration with IRAK-M-downregulated PBMCs.

To the best of our knowledge, the current study is the first to report that the administration of IRAK-M-downregulated PBMCs in septic mice activates splenic T cells and attenuates $\mathrm{T}$ cell apoptosis. Numerous studies have investigated $\mathrm{T}$ cell function in sepsis, and propose that apoptosis, cellular exhaustion, impaired responsiveness to stimuli and impaired cytokine responses are primarily involved $(18,21,22)$. In addition, previous studies have demonstrated that IL-7 treatment induces $\mathrm{T}$ cell proliferation and decreases lymphocyte apoptosis, thus improving the prognosis of patients diagnosed with sepsis (23-26). This is consistent with the results of the current study, as T cell apoptosis was decreased and the number of viable $T$ cells was increased following treatment with IRAK-M downregulated PBMCs. However, further studies investigating $\mathrm{T}$ cell function and phenotype diversity are required, as the results of the current study only provide a basis for further research on the use of IRAK-M downregulated PBMCs in sepsis.

In conclusion, the results of the current study demonstrate the direct effects of IRAK-M downregulation in PBMCs on the inflammatory response during sepsis in vitro and in vivo. The results indicate that IRAK-M serves an important role in the regulation of the immune response during sepsis, and provide a basis for further studies that will focus on the development of immunological therapies to treat patients with sepsis.

\section{Acknowledgements}

The authors would like to thank all the members of their laboratories, particularly Professor Shukun Fu at the Tenth People's Hospital (Shanghai, China) for their continuous support and guidance during the current study. The authors would also like to thank Dr Ming Wang from the Department of Statistics at Tongji University (Shanghai, China) and Mr. Jianjun Liu at Tongji University (Shanghai, China) for their encouragement.

\section{References}

1. Vincent JL, Opal SM, Marshall JC and Tracey KJ: Sepsis definitions: Time for change. Lancet 381: 774-775, 2013.

2. Ward PA: Immunosuppression in sepsis. JAMA 306: 2618-2619, 2011.

3. Li S, Strelow A, Fontana EJ and Wesche H: IRAK-4: A novel member of the IRAK family with the properties of an IRAK-kinase. Proc Natl Acad Sci USA 99: 5567-5572, 2002.

4. Zhou H, Yu M, Fukuda K, Im J, Yao P, Cui W, Bulek K, Zepp J, Wan Y,Kim TW, etal: IRAK-Mmediates Toll-likereceptor/IL-1Rinduced $\mathrm{NF \kappa B}$ activation and cytokine production. EMBO J 32: 583-596, 2013.

5. Wu Q, Jiang D, Smith S, Thaikoottathil J, Martin RJ, Bowler RP and Chu HW: IL-13 dampens human airway epithelial innate immunity through induction of IL-1 receptor-associated kinase M. J Allergy Clin Immunol 129: 825-833.e2, 2012.

6. Lin SC, Lo YC and Wu H: Helical assembly in the MyD88IRAK-4-IRAK-2 complex in TLR/IL-1R signalling. Nature 465: 885-890, 2010.

7. Deng L, Wang C, Spencer E, Yang L, Braun A, You J, Slaughter C, Pickart $\mathrm{C}$ and Chen ZJ: Activation of the IkappaB kinase complex by TRAF6 requires a dimeric ubiquitin-conjugating enzyme complex and a unique polyubiquitin chain. Cell 103: 351-361, 2000.

8. Escoll P, del Fresno C, García L, Vallés G, Lendínez MJ, Arnalich F and López-Collazo E: Rapid up-regulation of IRAK-M expression following a second endotoxin challenge in human monocytes and in monocytes isolated from septic patients. Biochem Biophys Res Commun 311: 465-472, 2003.

9. Deng JC, Cheng G, Newstead MW, Zeng X, Kobayashi K, Flavell RA and Standiford TJ: Sepsis-induced suppression of lung innate immunity is mediated by IRAK-M. J Clin Invest 116: 2532-2542, 2006.

10. Kobayashi K, Hernandez LD, Galán JE, Janeway CA Jr, Medzhitov R and Flavell RA: IRAK-M is a negative regulator of Toll-like receptor signaling. Cell 110: 191-202, 2002.

11. Monneret G, Venet F, Pachot A and Lepape A: Monitoring immune dysfunctions in the septic patient: A new skin for the old ceremony. Mol Med 14: 64-78, 2008.

12. Livak KJ and Schmittgen TD: Analysis of relative gene expression data using real-time quantitative PCR and the 2(-Delta Delta C(T)) method. Methods 25: 402-408, 2001. 
13. Marshall JC: Such stuff as dreams are made on: Mediator-directed therapy in sepsis. Nat Rev Drug Discov 2: 391-405, 2003.

14. Opal SM, Laterre PF, Francois B, LaRosa SP, Angus DC, Mira JP Wittebole X, Dugernier T, Perrotin D, Tidswell M, et al: Effect of eritoran, an antagonist of MD2-TLR4, on mortality in patients with severe sepsis: The ACCESS randomized trial. JAMA 309: 1154-1162, 2013

15. Hotchkiss RS and Karl IE: The pathophysiology and treatment of sepsis. N Engl J Med 348: 138-150, 2003.

16. Payen D, Monneret G and Hotchkiss R: Immunotherapy-a potential new way forward in the treatment of sepsis. Crit Care 17: 118, 2013.

17. Hall MW, Knatz NL, Vetterly C, Tomarello S, Wewers MD Volk HD and Carcillo JA: Immunoparalysis and nosocomial infection in children with multiple organ dysfunction syndrome. Intensive Care Med 37: 525-532, 2011.

18. Boomer JS, To K, Chang KC, Takasu O, Osborne DF, Walton AH, Bricker TL, Jarman SD II, Kreisel D, Krupnick AS, et al: Immunosuppression in patients who die of sepsis and multiple organ failure. JAMA 306: 2594-2605, 2011.

19. Wiersinga WJ, van't Veer C, van den Pangaart PS, Dondorp AM, Day NP, Peacock SJ and van der Poll T: Immunosuppression associated with interleukin-1R-associated-kinase-M upregulation predicts mortality in Gram

20. -negative sepsis (melioidosis). Crit Care Med 37: 569-576, 2009.

21. Restagno D, Venet F, Paquet C, Freyburger L, Allaouchiche B Monneret G, Bonnet JM and Louzier V: Mice survival and plasmatic cytokine secretion in a 'Two Hit' model of sepsis depend on intratracheal pseudomonas aeruginosa bacterial load. PLoS One 11: e0162109, 2016.
22. Patil NK, Bohannon JK and Sherwood ER: Immunotherapy: A promising approach to reverse sepsis-induced immunosuppression. Pharmacol Res 111: 688-702, 2016.

23. Boomer JS, Green JM and Hotchkiss RS: The changing immune system in sepsis: Is individualized immuno-modulatory therapy the answer? Virulence 5: 45-56, 2014.

24. Unsinger J, McGlynn M, Kasten KR, Hoekzema AS, Watanabe E, Muenzer JT, McDonough JS, Tschoep J, Ferguson TA, McDunn JE, et al: IL-7 promotes T cell viability, trafficking, and functionality and improves survival in sepsis. J Immunol 184: 3768-3779, 2010

25. Mackall CL, Fry TJ and Gress RE: Harnessing the biology of IL-7 for therapeutic application. Nat Rev Immunol 11: 330-342, 2011.

26. Kasten KR, Prakash PS, Unsinger J, Goetzman HS, England LG Cave CM, Seitz AP, Mazuski CN, Zhou TT, Morre M, et al: Interleukin-7 (IL-7) treatment accelerates neutrophil recruitment through gamma delta T-cell IL-17 production in a murine model of sepsis. Infect Immun 78: 4714-4722, 2010. 